\title{
Conservation and divergence of the genetic structure of larval foraging behaviour in two species of the Drosophila simulans clade
}

\author{
Raúl Godoy-Herrera ${ }^{1}$, Barrie Burnet ${ }^{2}$ and Kevin Connolly ${ }^{3}$ \\ ${ }^{1}$ Programa de Genética Humana, Instituto de Ciencias Biomédicas, Facultad de Medicina, Universidad de Chile, Independencia 1027, \\ Casilla 70061, Santiago-7, Chile; ${ }^{2}$ Department of Molecular Biology and Biotechnology, University of Sheffield, Sheffield S10 2UH, UK; \\ ${ }^{3}$ Department of Psychology, University of Sheffield, Sheffield S10 2TP, UK
}

\begin{abstract}
Larvae of the sibling species Drosophila simulans and $D$. mauritiana have rates of locomotor and feeding activity that are closely similar. Comparisons of the trait means for intraand interspecific hybrids show that significant epistatic interactions affect both characters when the genomes of the two species are combined. The phenotypic variances of
\end{abstract}

progenies obtained by backcrossing the interspecific hybrids to their respective parent species show that appreciable genetic turnover affecting foraging behaviour has occurred since their two phylogenetic lines diverged.

Heredity (2004) 92, 14-19, advance online publication, 24 September 2003; doi:10.1038/sj.hdy.6800356

Keywords: Drosophila; larval foraging; species hybrids; epistasis; coadaptation

\section{Introduction}

Three sibling species belonging to the melanogaster subgroup of the genus Drosophila are present as completely allopatric insular populations in the western Indian Ocean. Drosophila mauritiana is endemic to the island of Mauritius where it is the only species of the subgroup that is present. D. sechellia inhabits certain islands in the Seychelles group. D. simulans, widespread in central and eastern Africa is present in Madagascar, La Réunion and in the Seychelles, but it is not present on those islands occupied by $D$. sechellia (Lachaise et al, 1988). These three species are chromosomally homosequential and the adult insects are morphologically almost indistinguishable, apart from differences in the shape and size of the posterior lobe on the lateral plate of the male genital arch (Coyne, 1983). Estimates of the minimum number of quantitative trait loci underlying particular known species differences are summarised by Orr (2001).

With respect to the morphology of the posterior lobe, $D$. simulans and D. mauritiana are more different from each other than either is from D. melanogaster, whereas a multidimensional comparison based upon sexual behaviour, courtship songs and cuticular hydrocarbons shows that simulans and mauritiana are more closely similar to each other than either is to melanogaster (Cobb et al, 1989). There is asymmetrical sexual isolation between the species. Many of the genetic differences involved in sexual selection may have arisen since speciation has occurred (Carracedo et al, 2000). Lachaise et al (1986) cite

Correspondence: $R$ Godoy-Herrera, Programa de Genética Humana, Instituto de Ciencias Biomédicas, Facultad de Medicina, Universidad de Chile, Independencia 1027, Casilla 70061, Santiago-7, Chile.

E-mail: rgodoy@machi.med.uchile.cl

Received: 21 August 2002; revised: 20 May 2003; accepted: 10 July 2003 evidence suggesting that isolation is stronger between mauritiana and simulans from the Seychelles than it is from simulans originating elsewhere. Interspecific hybrid crosses give viable adult progeny of both sexes, consisting of fertile females and sterile males (Lachaise et al, 1986), so that viable progenies can be obtained from backcrosses to either of the parent species.

David et al (1989) consider that the ecological niche of a species is better defined by its larval breeding site than its adult feeding and mating sites. Their field investigations indicate that $D$. mauritiana is an abundant broad-niched opportunistic species breeding in sweet fermenting resources, with an ecological status similar to that of $D$. simulans. Larvae of species belonging to the simulans clade appear remarkably similar in their morphology, but Sucena and Stern (2000) have described a difference in the distribution of cuticular hairs caused by evolution of the cis-regulatory region of the ovo/ shaven-baby locus in the sechellia lineage. Thus far, no morphological differences between larvae of simulans and mauritiana, as far as we are aware, have been described, but it is note-worthy that the dentition posterior to the major cusp of the mouth hooks in D. mauritiana resembles that of $D$. sechellia rather than $D$. simulans (Muhammad-Ali and Burnet, unpublished).

The foraging behaviours of mauritania and simulans are similar, and both species have low tolerance to dietary ethanol compared with D. melanogaster (Muhammad-Ali and Burnet, 1995). D. melanogaster larvae exhibit adaptive behavioural responses during foraging. When searching for food their behaviour is characterised by increased locomotion with intermittent feeding movements. Where food is available, they shift to a pattern of trophic behaviour in which sustained feeding activity is associated with reduced locomotion (Green et al, 1983). Such behavioural flexibility could be adaptive in natural environments where there is patchy distribution of 
resources and localised accumulation of toxic or aversive metabolic by-products (Ruiz-Dubreuil et al, 1996).

Larval feeding rate is an important component of fitness. In populations of D. melanogaster it is stabilised at an intermediate optimum and is correlated with the ability to compete successfully for limited resources (Burnet et al, 1977; Ruiz-Dubreuil et al, 1996). Joshi and Mueller $(1988,1996)$ observed that mean feeding rate is higher in k-selected compared with r-selected populations and that there is density-dependent trade-off between food acquisition and food utilisation. Prasad et al (2001) also observed that selection for fast development time is associated with correlated changes in measures of foraging behaviour including feeding rate. Feeding rates also evolve in populations as correlated responses to selection on different larval characters, for example, in response to selection for increased parasitoid resistance (Fellowes et al, 1999), or to accumulation of metabolic by-products in the larval food (Borash et al, 2000). While these studies focus on D. melanogaster in particular, such relationships could provide useful indications of what may be expected in other species of the subgroup, such as D. simulans and D. mauritiana, which have comparable status as scramble-type competitors and which appear to occupy similar ecological niches.

Similarity in ecological status of simulans and mauritania larvae together with the quantitative phenotypic similarity of the main components of their foraging activities on a live yeast substrate (Muhammad-Ali and Burnet, 1995) might imply that only a limited genetic divergence has taken place between them. In this investigation we examine this possibility. An indication that divergence has occurred can be had by examining behaviour for evidence of hybrid breakdown such as has been detected in hybrids between two neotropical species of Drosophila by Godoy-Herrera et al (1994). Larvae show age-related change in locomotion and feeding between first and second instar (Burnet et al, 1977; Godoy-Herrera et al, 1984). Larvae of both stages are examined here in order to find whether epigenetic change in behaviour occurs in the hybrids. Larvae were also observed under two conditions with and without food. This was to ascertain if hybrid larvae exhibit behavioural flexibility in modulating their rates of feeding and locomotion in response to environmental change.

We also compare the phenotypic variances of hybrid larvae with those derived from backcrosses to their respective parent species. The morphology of the posterior process on the male genital arch provides us with a character that has diverged between the species and we use this as a comparator against which to assess the magnitude of proportional differences in the phenotypic variances of the interspecific hybrids and backcrosses for larval behaviour.

\section{Materials and methods}

D. simulans originating from Nairobi, Kenya and from the Seychelles, also D. mauritiana G73 and G93 from Mauritius in the Indian Ocean were derived from wild populations. They were obtained from the stock collection of Laboratoire de Biologie et Génétique Evolutives, CNRS, Gif sur Yvette, France, and have been maintained in mass cultures for several years before observations of larval behaviour began. The stocks were maintained under moderate crowding density on oatmeal/molasses agar medium seeded liberally with live bakers' yeast, at a constant temperature of $25^{\circ} \mathrm{C}$ and standard lighting schedule of $12 \mathrm{~h}$ light and $12 \mathrm{~h}$ dark.

Virgin flies were collected from low-density cultures at $3 \mathrm{~h}$ intervals and stored on freshly yeasted food medium. Virgin flies were mated in groups. Approximately equalsized groups of females were used for egg collections for parental, intraspecific and interspecific crosses.

Groups of females aged 3-5 days were allowed to oviposit for $3 \mathrm{~h}$ on a layer of live baker's yeast over water agar. The eggs were incubated at $25^{\circ} \mathrm{C}$ and newly hatched larvae transferred at low-density to freshly yeasted vials. Specified larval ages are given in hours after hatching from the egg.

Using a stereomicroscope, each larva was observed in a $9 \mathrm{~cm}$ Petri dish containing a layer of water-agar gel. The substrate conditions were: (i) no food - water-agar gel $3 \%$, or (ii) food - agar gel overlaid with a suspension of fresh live baker's yeast at $48 \% \mathrm{w} / \mathrm{v}$.

Behavioural observations were recorded and analysed using a multichannel event recorder based on a Toshiba T1200 laptop computer, following the procedure described in detail by Ruiz-Dubreuil et al (1996). Feeding activity was measured as the number of scooping movements per minute of the mouth hooks and cephalopharyngeal apparatus. Locomotion was measured as the number of sequential contractions per minute passing in series along the body (Green et al, 1983). These two measures of behaviour, which are both measures of activity, are expressed as unit counts of body movements, which seem of direct relevance to energetic costs. A different measure of locomotion, 'path length' (Sokolowski et al, 1997) appears to be a compound measure which contains at least two distinguishable components - locomotor activity per se and vagility - the significance of which is discussed by Ruiz-Dubreuil et al (1996). The two measures of locomotion are related, but only the former is used here.

Observations were made on the behaviour of early first instar (3-5 h old) larvae of $D$. simulans and D. mauritiana (two stocks of each species), their respective reciprocal $F_{1}$ intraspecific hybrids and four of the possible $F_{1}$ interspecific hybrids (see Table 1). In total, 50 larvae were observed from each of the 12 genotype groups and each larva was observed in yeast suspension for a period of $2 \mathrm{~min}$. One larva from every genotype group, selected in random order, was observed at each recording session, so that any possible environmental variables or age differences were orthogonal to time.

For the comparison of larval behaviour of $\mathrm{F}_{1}$ interspecific hybrids with the progeny of the respective backcrosses (Table 4) larvae of all three genotype groups were observed in the same generation. Virgin $F_{1}$ interspecific hybrid females derived from matings between simulans (Nairobi) females and mauritiana (G71) males were backcrossed to males of the respective parent species. A contemporary cross between the parent species was then repeated to generate a new $F_{1}$ so that larvae of all three genotype groups were available at the same time. Second instar larvae $(48-50 \mathrm{~h}$ old) were each observed for $5 \mathrm{~min}$ in yeast suspension and then 
immediately after for $5 \mathrm{~min}$ on agar without food. In total, 50 larvae were observed from each group.

The external genitalia of adult male flies were dissected under Ringer's solution. The relevant anatomical details and species differences are well illustrated by True et al (1997). The lateral plates from the left and right sides of the genital arch were dissected and spread in a horizontal plane and mounted in Berlese fluid under a glass coverslip. Each pair of lateral plates was photographed at $\times 60$ magnification. Measurements of the perimeter and area of the posterior lobe on each lateral plate were made electronically from the photographic prints using a scanner. Choice of the best straight line projection separating the profile of each posterior lobe from the lateral plate was made by eye and to that extent is subjective. However, repeated measurements showed that consistency and agreement between observers were achieved. Measurements from the left and right sides of each fly were averaged. In all, 20 males were measured from each genotype group. Results presented in Table 5 are in arbitrary units.

\section{Results}

\section{Larval behaviour}

Table 1 summarises the mean expression of locomotor and feeding activity of 3-5-h-old first instar larvae observed while foraging on a lawn of live yeast. The 12 genotype groups consist of two stocks of each of two parental species: D. simulans (Nairobi and Seychelles) and D. mauritiana (G71 and G93), their four respective $F_{1}$ reciprocal intraspecific hybrids and four of the possible $F_{1}$ interspecific hybrids. The larvae were synchronised to the same age and observed under identical environmental conditions.

\section{Locomotor activity}

Analysis of variance (ANOVA) for locomotor activity indicates that there are significant differences over the 12 genotype groups, $\mathrm{F}_{11,599}=6.1, P<0.01$. The larvae of $D$. simulans (Nairobi) have significantly lower rates of

Table 1 Behaviour of early first instar larvae of D. simulans, $D$. mauritiana and their intraspecific and interspecific hybrids

\begin{tabular}{lll}
\hline & Locomotion & Feeding rate \\
\hline D. simulans Nairobi & $15.90 \pm 1.28$ & $56.40 \pm 2.21$ \\
Seychelles & $21.80 \pm 1.39$ & $52.50 \pm 2.27$ \\
$\mathrm{~F}_{1}$ Nai. $\times$ Sey. & $20.80 \pm 1.34$ & $56.80 \pm 2.65$ \\
$\mathrm{~F}_{1}$ Sey. $\times$ Nai. & $20.70 \pm 1.84$ & $56.00 \pm 2.61$ \\
D. mauritiana 93 & $23.90 \pm 1.95$ & $58.60 \pm 2.95$ \\
71 & $21.30 \pm 1.23$ & $54.70 \pm 2.57$ \\
$\mathrm{~F}_{1} 93 \times 71$ & $27.70 \pm 1.65$ & $53.30 \pm 3.75$ \\
$\mathrm{~F}_{1} 71 \times 93$ & $28.40 \pm 1.54$ & $56.90 \pm 3.97$ \\
Interspecific hybrids & & \\
$\mathrm{F}_{1}$ Nai. $\times 71$ & $22.80 \pm 1.12$ & $46.20 \pm 2.54$ \\
$\mathrm{~F}_{1} 71 \times$ Nai. & $20.80 \pm 1.52$ & $45.00 \pm 2.53$ \\
$\mathrm{~F}_{1}$ Sey. $\times 93$ & $27.70 \pm 1.70$ & $32.90 \pm 2.04$ \\
$\mathrm{~F}_{1} 93 \times$ Sey. & $24.30 \pm 1.33$ & $44.90 \pm 2.59$ \\
\hline
\end{tabular}

Larvae were observed on a lawn of live baker's yeast. Feeding rate is expressed as the mean \pm SE per minute of the number of movements made by the larval mouth hooks, and locomotion as the number of segmental contractions per minute. For all crosses, the usual convention is followed: the first parent shown is the female.
Table 2 Comparison of the mean locomotor activity of (a) intraspecific hybrids with the parental strains of each species, and (b) the means of the interspecific with those of the intraspecific hybrids (see Table 1)

\begin{tabular}{lcllll}
\hline (a) Intraspecific hybrids vs parents & & & \\
& \multicolumn{2}{c}{ simulans } & & & mauritiana \\
\cline { 2 - 3 } \cline { 5 - 6 } & Nai. & Sey. & & 71 & 93 \\
\hline Nai. $\times$ Sey & $*(+)$ & $\bullet$ & & \\
Sey. $\times$ Nai. & $*(+)$ & $\bullet$ & & $*(+)$ & $*(+)$ \\
$71 \times 93$ & & & & $*(+)$ & $*(+)$ \\
$93 \times 71$ & & & &
\end{tabular}

(b) Interspecific hybrids vs intraspecific hybrids

\begin{tabular}{lccccc} 
& \multicolumn{2}{c}{ simulans } & & \multicolumn{2}{c}{ mauritiana } \\
\cline { 2 - 3 } & Nai. $\times$ Sey. & Sey $\times$ Nai. & & $93 \times 71$ & $71 \times 93$ \\
\hline Nai. $\times 71$ & $\bullet$ & $\bullet$ & $*(-)$ & $*(-)$ \\
$71 \times$ Nai. & $\bullet$ & $\bullet$ & & $*(-)$ & $*(-)$ \\
Sey. $\times 93$ & $*(+)$ & $*(+)$ & & $\bullet$ & $*$ \\
$93 \times$ Sey. & $*(+)$ & $*(+)$ & $*(-)$ & $*(-)$ \\
\hline
\end{tabular}

*Denotes differences significant to at least the $5 \%$ level of probability. - identifies differences that are not significant. Signs in brackets indicate the direction of the deviation of the means of intraspecific hybrids from those of their respective parents or of the interspecific hybrids from the intraspecific hybrids.

locomotion than those of the Seychelles stock. The two strains of D. mauritiana do not differ significantly from one another or from the Seychelles strain of $D$. simulans. Relevant comparisons of the locomotor activity between parent species stocks and their respective reciprocal intraspecific hybrids are summarised in Table 2a.

The two $F_{1}$ intraspecific hybrid groups of $D$. simulans do not differ significantly from one another or from their Seychelles parents but both reciprocal cross progenies have significantly higher mean locomotor activity scores than larvae of the Nairobi strain. Evidently, there is some degree of inbreeding depression for this character in the Nairobi stock.

Both groups of reciprocal $F_{1}$ intraspecific hybrids of D. mauritiana have significantly higher mean locomotor activity scores than either of the parent strains. Such heterosis for locomotor activity infers a degree of inbreeding depression for the character within both of the parent stocks of D. mauritiana.

Inbreeding depression results from random fixation of alleles conferring lower expression of the character and reduced fitness. Intraspecific hybrids are heterozygous at loci affecting larval locomotion for which the parent strains differ and the overall level of multilocus heterozygosity is consequently closer to that in a larger panmictic population. The intraspecific hybrids rather than the parental strains seem to provide a more suitable comparator against which to assess the mean levels of performance of the progeny of the interspecific hybrid crosses.

The comparisons of mean scores between intra- and interspecific hybrids summarised in Table $2 \mathrm{~b}$ present an interesting array of contrasting outcomes. The reciprocal progenies derived from crosses between $D$. simulans (Nairobi) and D. mauritiana (G71) have similar levels of locomotor activity. They are not significantly different from those of $D$. simulans but, in both instances, they 
are significantly different from those of $D$. mauritiana. The interspecific hybrid progenies of crosses between D. simulans (Seychelles) and D. mauritiana (G93) have significantly higher mean locomotor activity scores than $D$. simulans but for one reciprocal it is significantly lower than D. mauritiana. That is, there is prima facie evidence for both heterosis and hybrid breakdown affecting larval locomotion depending upon which particular interspecific hybrid genetic background one is considering.

\section{Feeding activity}

Larval feeding rate scores are shown in Table 1. ANOVA indicates that there are significant differences among genotype groups, $\mathrm{F}_{11,599}=7.24, P<0.01$. The reciprocal intraspecific hybrids of both species are similar to, and not significantly different from, the parent strains, whereas differences between the intra- and interspecific hybrids are significant, $F_{1}, 588=45.9, P<0.01$. The deviation of the mean score of each group of interspecific hybrids is in every comparison negative and significant at $P<0.05$ (Table 3 ). The reduced feeding rates constitute substantial evidence of hybrid breakdown for the character in the interspecific hybrid larvae.

Evidence for hybrid breakdown in interspecific hybrids, such as that presented above, suggests that when the genomes of different species are combined some degree of genetic imbalance is created in the coadapted gene complexes which regulate expression of larval behaviours at an adaptive norm. Progenies arising from backcrosses of the interspecific hybrids to their parent species might be used to test for any additional effects of segregation and recombination such as may affect allelic interactions within and between loci in the hybrid genomes.

Table 4 shows the mean locomotor activity and rates of feeding for larvae of the $F_{1}$ progeny of the cross between the Nairobi strain of $D$. simulans and G71 strain of $D$. mauritiana. Second instar larvae of backcross progenies were obtained from matings between interspecific hybrid females and males of the respective parent species. Larvae at this stage of development do not differ significantly for either locomotor or feeding activity (cf. Table 1). Feeding rate scores are also higher than in the first instar.

For larvae observed in yeast suspension the mean scores for locomotor activity of the backcross are a little lower than for the interspecific hybrids but the difference is not significant $\left(\mathrm{F}_{2}, 147=1.53, P=0.2\right)$. The phenotypic variance is relatively greater among the backcross progeny, which is indicated by comparing the coefficients of variation of the groups. Relative to the $F_{1}$ it

Table 3 Comparisons of the mean rates of larval feeding activity for interspecific $v s$ intraspecific hybrids (see Table 1)

\begin{tabular}{lccccc}
\hline & \multicolumn{2}{c}{ simulans } & & \multicolumn{2}{c}{ mauritiana } \\
\cline { 2 - 3 } \cline { 5 - 6 } & Nai. $\times$ Sey. & Sey.$\times$ Nai. & & $93 \times 71$ & $71 \times 93$ \\
\hline Nai. $\times 71$ & $*(-)$ & $*(-)$ & & $*(-)$ & $*(-)$ \\
$71 \times$ Nai. & $*(-)$ & $*(-)$ & & $*(-)$ & $*(-)$ \\
Sey. $\times 93$ & $*(-)$ & $*(-)$ & & $*(-)$ & $*(-)$ \\
$93 \times$ Sey. & $*(-)$ & $*(-)$ & & $*(-)$ & $*(-)$ \\
\hline
\end{tabular}

The key to symbols is shown in Table 2. increased $38 \%$ in the backcross to simulans and $43 \%$ in the backcross to mauritiana.

There is also a small but not significant reduction in mean feeding rate in both backcrosses $\left(\mathrm{F}_{2}, 147=1.24\right.$, $P=0.3)$, associated with an increase in variability. Relative to the $\mathrm{F}_{1}$ the coefficients of variation increased $70 \%$ in the backcross to simulans and $23 \%$ in the backcross to mauritiana.

On moist water agar without food larvae showed a more than two-fold increase in locomotor activity. This increase occurs in all three genotype groups, but the means for the backcrosses are significantly lower than in the $F_{1}\left(F_{2}, 147=3.92, P=0.02\right)$. Relative to the $F_{1}$ the coefficients of variation for locomotion increased $58 \%$ in the backcross to mauritiana.

Larvae moving over moist agar performed cephalopharyngeal movements corresponding to feeding, but at a mean rate approximately half that observed in yeast suspension. There are no significant differences between the means of the three genotype groups, but the coefficients of variation increased in the backcross to simulans $17 \%$ and $64 \%$ in the backcross to mauritiana.

\section{Genital arch morphology}

The size of the posterior lobe on the genital arch, measured in terms of the perimeter and area, shown in Table 5, clearly distinguishes between males of $D$. simulans and D. mauritiana. The midparent values for both measures (perimeter 7.52 and area 2.74) are close to those for the $\mathrm{F}_{1}$ interspecies hybrids and the backcross means are intermediate between the $F_{1}$ and those of males of their respective species, pointing to a largely additive pattern of inheritance for both measures of posterior lobe size. There is an appreciable expansion of the phenotypic variances in the backcrosses. The coefficient of variation for the perimeter in the backcross to simulans increased $311 \%$ and in the backcross to mauritiana $239 \%$ relative to the $\mathrm{F}_{1}$. For the area measure, the coefficient of variation in the backcross to simulans increased $590 \%$ and in the backcross to mauritiana $606 \%$.

\section{Discussion}

Larval foraging behaviour is affected by the environment (Green et al, 1983; Godoy-Herrera et al, 1994). Larvae observed in yeast have a relatively low rate of locomotion and feed rapidly. In the absence of food an increase in locomotion accompanies reduced frequency of mouth hook movements which occur as repeated short bursts instead of a continuous rhythm. The larvae also exhibit epigenetic changes, that is, the frequencies of behaviours alter according to larval age (Green et al, 1983; GodoyHerrera et al, 1984). Although the trajectories of change are not the same, for species and strains within species, there is a tendency for feeding rates to increase as growth proceeds from eclosion until the middle of the third instar. Expression of epigenetic change as between the first and second instar, and the flexibility of behavioural responses in the second instar, appear to remain intact in the interspecific and introgressive (backcross) hybrids between $D$. simulans and D. mauritiana. Consequently, canalisation of the structure of foraging behaviours within their phenotypic norms of reaction is not disrupted in the hybrids. Further 
Table 4 Behaviour of second instar larvae from a cross between D. simulans (Nairobi) and D. mauritiana (G71) compared with larvae from reciprocal backcrosses to the parent species

\begin{tabular}{|c|c|c|c|c|c|c|}
\hline & \multicolumn{2}{|c|}{$F_{1}$} & \multicolumn{4}{|c|}{ Backcross to } \\
\hline & sim..$+\times$ mau. ô $^{-}$ & $c . v$ & simulans $\widehat{0}$ & c.v. & mauritiana $\widehat{o}$ & c.v. \\
\hline \multicolumn{7}{|l|}{ Yeast } \\
\hline Locomotion & $29.40 \pm 1.81$ & 5.57 & $25.00 \pm 1.96$ & 7.70 & $25.60 \pm 2.02$ & 7.99 \\
\hline Feeding & $162.00 \pm 7.47$ & 17.30 & $144.00 \pm 9.21$ & 29.50 & $156.00 \pm 8.15$ & 21.30 \\
\hline \multicolumn{7}{|l|}{ Agar } \\
\hline Locomotion & $69.50 \pm 2.72$ & 5.32 & $57.00 \pm 3.56$ & 11.10 & $61.50 \pm 3.21$ & 8.40 \\
\hline Feeding & $86.70 \pm 2.19$ & 2.76 & $87.50 \pm 2.38$ & 3.23 & $85.10 \pm 2.77$ & 4.53 \\
\hline
\end{tabular}

Behaviours are expressed as the mean \pm SE events per minute. c.v. denotes the coefficient of variation (var/mean).

Table 5 Size of the posterior lobe on the lateral plate of the genital arch of males of the two parent species, interspecific hybrids and of the reciprocal backcross progenies

\begin{tabular}{|c|c|c|c|c|}
\hline & \multicolumn{2}{|c|}{ Perimeter } & \multicolumn{2}{|c|}{ Area } \\
\hline & $M e a n \pm S E$ & c.v. & $M e a n \pm S E$ & c.v. \\
\hline D. simulans & $10.70 \pm 0.12$ & 0.03 & $6.52 \pm 0.12$ & 0.05 \\
\hline D. mauritiana & $4.34 \pm 0.05$ & 0.01 & $0.77 \pm 0.02$ & 0.01 \\
\hline $\mathrm{F}_{1}\left(\operatorname{sim} . q \times\right.$ mau. $\left.0^{\hat{0}}\right)$ & $7.16 \pm 0.10$ & 0.03 & $2.94 \pm 0.07$ & 0.03 \\
\hline Backcross to sim. $\delta^{t}$ & $8.85 \pm 0.23$ & 0.12 & $4.69 \pm 0.22$ & 0.21 \\
\hline Backcross to mau. $\hat{0}$ & $5.37 \pm 1.20$ & 0.10 & $1.51 \pm 0.12$ & 0.22 \\
\hline
\end{tabular}

quantitative comparisons using transition analysis could be used to test this more rigorously.

Inbreeding depression affected the locomotor activity of first instar larvae in the parent strains of both species, but there was no corresponding evidence for it with respect to feeding rate. It appears to be attributable to the fixation of different recessive alleles with specific effects upon locomotion as distinct from more generalised effects upon neuromotor functions. Of greater interest is evidence for positive epistasis affecting larval locomotion in one set of interspecific hybrids and negative epistasis in another. Positive and negative in this context refer only to the direction of the deviation in expression of the character. On the assumption that the fitness optimum is at, or close to, the intermediate mean phenotype, deviations in either direction, whether due to epistasis or not, are likely to be correlated with negative effects on fitness. Evidence of negative heterosis is particularly strong for feeding rate. It is consistent over the array of interspecific hybrids examined here. These epistatically interacting allelic substitutions affecting foraging behaviour have probably occurred since the phylogenies of $D$. simulans and $D$. mauritiana diverged.

Progenies obtained by backcrossing interspecific hybrids to either $D$. simulans or $D$. mauritiana have similar mean phenotype scores to those for the $F_{1}$ itself, except in the case of locomotion out of food, where the scores are significantly lower. Increases in phenotypic variance in both backcrosses are due to recombination at gene loci for which the parent species differ. The variation for both behavioural characters is constrained compared with that for the size of the posterior lobe of the male genital arch in the same genotype groups, which has a largely additive pattern of inheritance (Liu et al, 1996; Zeng et al, 2000). The variances of backcross progenies should, of course, increase as the trait means in the parent species diverge. Trait means for the behavioural characters have not diverged to any significant extent, but there is more to it than that.

The conclusion that selection for larval feeding rate is generally stabilising is confirmed by the decline in feeding rates shown by populations moved from crowded to uncrowded environments (Mueller and Joshi, 2000). Characters under stabilising selection show 'genetic turnover' (Orr, 2001), that is, genes affecting the trait continue to diverge even after trait means have stabilised (Lande, 1975; Barton, 1989). In the particular case of larval feeding rate, the population means for simulans and mauritiana are similar at synchronous stages in larval development, and presumably also close to the respective fitness optima of each species. According to Turelli et al (2001), during 0.5 Myr every locus on average could have undergone one nonsynonymous base pair substitution (Orr and Turelli, 2001). This period accords with the approximate range of estimates of the time from which divergence of the phylogenies of simulans and mauritiana took place (Ashburner et al, 1984; Hey and Kliman, 1993). Not all nonsynonymous substitutions can have consequences for phenotypic expression of the character and those that do are, necessarily, a balanced assembly with individually positive or negative effects on the mean phenotype.

Many characters that contribute to postzygotic reproductive isolation between species show evidence of epistasis (Wu and Palopoli, 1994; Johnson, 2000; Orr, 2001). Our results extend this evidence to larval characters. Pointing to the large number of 'hybrid sterility genes' so far detected within the D. melanogaster subgroup, Johnson (2000) concludes that, despite minimal morphological divergence between species, substantial functional divergence has occurred. With regard to the two species of the $D$. simulans clade described here it seems that, although quantitative expression of larval foraging has been conserved, genetic divergence in the control of this behaviour has taken place.

\section{Acknowledgements}

We thank Monica Kibart for technical assistance. This work was supported by a grant (RSG/176) from the University of Sheffield Research Fund. We are grateful to The Royal Society, London, and The British Council, Santiago, for enabling exchange visits between Chile and the UK. 


\section{References}

Ashburner M, Bodmer M, Lemeunier F (1984). On the evolutionary relationships of Drosophila melanogaster. Dev Genet 4: 295-312.

Barton NH (1989). The divergence of a polygenic system under stabilising selection, mutation and drift. Genet Res 54: 59-77.

Borash DJ, Teotonio H, Rose MR, Mueller LD (2000). Densitydependent natural selection in Drosophila: correlations between feeding rate, development time, and viability. J Evol Biol 13: 181-187.

Burnet B, Sewell D, Bos M (1977). Genetic analysis of larval feeding behaviour in Drosophila melanogaster. II. Growth relations and competition between selected lines. Genet Res 30: 149-161.

Carracedo MC, Suarez C, Casares P (2000). Sexual isolation between Drosophila melanogaster, D. simulans and D. mauritiana: sex and species specific discrimination. Genetica 108: 155-162.

Cobb M, Burnet B, Blizard R, Jallon J-M (1989). Courtship in Drosophila sechellia: its structure, functional aspects, and relationships to those of other members of the members of the Drosophila melanogaster species subgroup. J Insect Behav 2: $63-89$.

Coyne JA (1983). Genetic basis of differences in gential morphology among three sibling species of Drosophila. Evolution 37: 1101-1118.

David JR, McEvey SF, Solignac M, Tsacas L (1989). Drosophila communities on Mauritius and the ecological niche of $D$. mauritiana (Diptera: Drosophilidae). J Afr Zool 103: 107-116.

Fellowes MDE, Kraaijeveld AR, Godfray HCJ (1999). Association between feeding rate and parasitoid resistance in Drosophila melanogaster. Evolution 53: 1302-1305.

Godoy-Herrera R, Burnet B, Connolly K, Gogarty J (1984). The development of locomotor activity in Drosophila melanogaster larvae. Heredity 52: 63-75.

Godoy-Herrera R, Burnet B, Connolly K, Grey D, Weir I (1994). Disruption of the structure of larval foraging behaviour in interspecific hybrids in Drosophila. Heredity 72: 260-268.

Green CH, Burnet B, Connolly KJ (1983). Organization and patterns of inter- and intraspecific variation on the behaviour of Drosophila larvae. Anim Behav 31: 282-291.

Hey J, Kliman RM (1993). Population genetics and phylogenetics of DNA sequence variation at multiple loci within the Drosophila melanogaster species complex. Mol Biol Evol 10: 804-822.

Johnson NA (2000). Gene interactions and the origin of species. In: Wolf JB, Brodie ED, Wade MJ (eds) Epistasis and the Evolutionary Process, Oxford University Press Inc.: New York. pp 197-212.

Joshi A, Mueller LD (1988). Evolution of higher feeding rate in Drosophila due to density-dependent natural selection. Evolution 42: 1090-1093.
Joshi A, Mueller LD (1996). Density-dependent natural selection in Drosophila: trade-offs between larval food acquisition and utilization. Evol Ecol 10: 463-474.

Lachaise D, Cariou M-L, David JR, Lemeunier F, Tsacas L, Ashburner M (1988). Historical biogeography of the Drosophila melanogaster species subgroup. In: Hecht MK, Wallace B, Prance GT (eds) Evolutionary Biology, Plenum Press: New York. Vol 22 pp 159-225.

Lachaise D, David JR, Lemeunier F, Tsacas L (1986). The reproductive relationships of Drosophila sechellia with $D$. mauritiana, D. simulans, and D. melanogaster from the Afrotropical region. Evolution 40: 262-271.

Lande R (1975). The maintenance of genetic variability by mutation in a polygenic character with linked loci. Genet Res 26: 221-235.

Liu J, Mercer JM, Stam LF, Gibson GC, Zeng Z-B, Laurie CC (1996). Genetic analysis of a morphological shape difference in the male genitalia of Drosophila simulans and D. mauritiana. Genetics 142: 1129-1145.

Mueller LD, Joshi A (2000). Stability in Model Populations. Princeton University Press: Princeton, NJ.

Muhammad-Ali AZ-K, Burnet B (1995). Ethanol tolerance and variation at the alcohol dehydrogenase (Adh) locus of Drosophila mauritiana. Heredity 74: 438-444.

Orr HA 2001. The genetics of species differences. Trends Ecol Evol 16: 343-350.

Orr HA, Turelli M (2001). The evolution of postzygotic isolation: accumulating Dobzhansky-Muller incompatibilities. Evolution 55: 1085-1094.

Prasad NG, Shakarad M, Anitha D, Rajamani M, Joshi A (2001). Correlated responses to selection for faster development and early reproduction in Drosophila: the evolution of larval traits. Evolution 55: 1363-1372.

Ruiz-Dubreuil G, Burnet B, Connolly K, Furness P (1996). Larval foraging behaviour and competition in Drosophila melanogaster. Heredity 76: 55-64.

Sokolowski MB, Pereira HS, Hughes K (1997). Evolution of foraging behavior in Drosophila by density-dependent selection. Proc Nat Acad Sci USA 94: 7373-7377.

Sucena E, Stern DL (2000). Divergence of larval morphology between Drosophila sechellia and its sibling species caused by cis-regulatory evolution of ovo/shaven-baby. Proc Natl Acad Sci USA 97: 4530-4534.

True JR, Liu J, Stam LF, Zeng Z-B, Laurie CC (1997). Quantitative genetic analysis of divergence in male secondary sexual traits between Drosophila simulans and Drosophila mauritiana. Evolution 51: 816-832.

Turelli M, Barton N, Coyne JA (2001). Theory and speciation. Trends Ecol Evol 16: 330-342.

Wu C-I, Palopoli MF (1994). Genetics of postmating reproductive isolation in animals. Ann Rev Genet 27: 283-308.

Zeng Z-B, Liu J, Stam LF, Kao C-H., Mercer JM, Laurie CC (2000). Genetic architecture of a morphological shape difference between two Drosophila species. Genetics 154: 299-310. 